\title{
Iterative learning control of a membrane deformable mirror for optimal wavefront correction
}

\author{
Aleksandar Haber, ${ }^{1, *}$ Alessandro Polo, ${ }^{2}$ Carlas S. Smith, ${ }^{1}$ Silvania F. Pereira, ${ }^{2}$ \\ Paul Urbach, ${ }^{2}$ and Michel Verhaegen ${ }^{1}$ \\ 'Delft Center for Systems and Control, Delft University of Technology, Mekelweg 2, Delft 2628 CD, The Netherlands \\ ${ }^{2}$ Optics Research Group, Delft University of Technology, Lorentzweg 1, Delft 2628 CJ, The Netherlands \\ *Corresponding author: a.haber@tudelft.nl
}

Received 23 January 2013; accepted 21 February 2013;

posted 4 March 2013 (Doc. ID 183576); published 10 April 2013

\begin{abstract}
We present an iterative learning control (ILC) algorithm for controlling the shape of a membrane deformable mirror (DM). We furthermore give a physical interpretation of the design parameters of the ILC algorithm. On the basis of this insight, we derive a simple tuning procedure for the ILC algorithm that, in practice, guarantees stable and fast convergence of the membrane to the desired shape. In order to demonstrate the performance of the algorithm, we have built an experimental setup that consists of a commercial membrane DM, a wavefront sensor, and a real-time controller. The experimental results show that, by using the ILC algorithm, we are able to achieve a relatively small error between the real and desired shape of the DM while at the same time we are able to control the saturation of the actuators. Moreover, we show that the ILC algorithm outperforms other control algorithms available in the literature. (C) 2013 Optical Society of America

OCIS codes: $\quad$ (110.1080) Active or adaptive optics; (090.1000) Aberration compensation; (230.3990)

Micro-optical devices; (150.5495) Process monitoring and control.

http://dx.doi.org/10.1364/AO.52.002363
\end{abstract}

\section{Introduction}

Adaptive Optics (AO) is a well-established technique for correcting wavefront aberrations in optical systems. The basic principle of $\mathrm{AO}$ is to measure wavefront aberrations using a wavefront sensor (WFS) and to compensate them by changing the geometry of an active optical element in the system. One of the first successful applications of the AO technique was in astronomy $[\underline{1}, 2]$ to compensate wavefront aberrations induced $\overline{b y}$ atmospheric turbulence. Nowdays, AO technique is used in the fields of

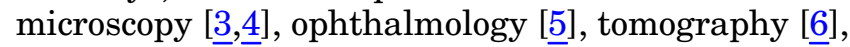
laser beam shaping [7], optical communication [8] and more recently in lithography [9-11].

$1559-128 \mathrm{X} / 13 / 112363-11 \$ 15.00 / 0$

(C) 2013 Optical Society of America
The basic components of an AO system are WFS, an active optical element like a deformable mirror (DM) or a spatial light modulator, and a control algorithm. There are different types of DMs. Most widely used are segmented, microelectromechanical systems, bimorph piezoelectric, and membrane DMs. Membrane DMs are relatively cheap, they have low hysteresis, and they have low power consumption. However, the response of membrane mirrors is nonlinear and the coupling between control channels is relatively strong [12].

In the literature various modeling and control strategies for membrane DMs have been proposed [12-14]. The control strategy presented in [12] is based on a steepest-descent optimization algorithm. The control strategy presented in [14] is derived by inverting an influence function (matrix) of a DM. In [13], the problem of controlling a membrane DM is formulated as a non-negative least squares (NNLS) problem. However, the tuning of the parameters of the above cited 
control strategies is performed empirically, by trial and error. Because of this, these control strategies might not guarantee optimal performance of an AO system. This nonoptimality manifests itself in the slow convergence of the wavefront error and in a significant steady-state wavefront error. The nonoptimality of the above cited control strategies also originates from a somewhat heuristic method used to identify a DM model (an influence matrix). Namely, in the above cited papers, the $i$ th column of an influence matrix is a measurement of a membrane response when the step voltage is applied to the $i$ th channel. Although simple, this identification method directly incorporates measurement errors into a DM model. Furthermore, this method does not take into account the "cross-talk" between the channels of a DM. Another drawback of the above cited control strategies is that they do not properly address the problem of saturation of the actuators of a DM. The saturation of the actuators is an undesired phenomena. On the one hand, it creates a strong mechanical stress on the membrane of a DM, and thus it reduces its lifetime. On the other hand, saturation might prevent undamped control algorithms to converge.

In order to boost the performance of $\mathrm{AO}$ systems, in this paper we present an iterative learning control (ILC) algorithm for controlling the shape of a membrane DM. The presented ILC algorithm is based on the linearized model of the DM, that is identified from the experimental data. We furthermore give a physical interpretation of the design parameters of the ILC algorithm. On the basis of this insight, we derive a simple tuning procedure for the ILC algorithm that, in practice, guarantees stable and fast convergence of the membrane to the desired shape. In order to demonstrate the performance of the new algorithm we have built an experimental setup that consists of a commercial membrane DM, a WFS, and a real-time controller. The experimental results show that by using this new ILC algorithm we are able to achieve a relatively small error between the real and desired shape of the DM while at the same time we are able to control the saturation of the actuators. Moreover, we show that the ILC algorithm outperforms other control algorithms available in the literature. We also present a simple and effective statistical identification procedure for a linearized model of a DM. Using this model, and only one initial measurement of the DM shape, we can apply the ILC algorithm offline. After the ILC algorithm has converged, we apply a "learned input" to a DM. Because it uses only one measurement, this is a fast method for generating the desired shape.

The benefits of the ILC algorithm for correcting wavefront aberrations were first demonstrated in [15]. However, in [15] the ILC algorithm only penalizes the differences between the voltages of two consecutive controliterations. Although this approach enables us to control the convergence rate of the ILC algorithm, in some situations the steady-state voltages can still saturate. Furthermore, because the convergence and stability of the ILC algorithm have not been studied in [15], the tuning of the parameters of the ILC algorithm have to be performed online on the real setup which can be time consuming. In contrast to [15], in this paper we penalize the values of the voltages for the next control iteration which enable us to directly control the voltage saturation. Beside this, we perform the stability and convergence analysis of the ILC algorithm and we propose a simple tuning procedure which can be performed offline.

This paper is organized as follows. In Section 2, we describe an experimental setup. In Section $\underline{3}$, we present an ILC control algorithm. In Section 4, we present an identification procedure for a model of the DM. In Section 5, we present experimental results and in Section 6, we draw conclusions.

\section{Experimental Setup}

In this section we describe the optical test bench (AO system) that we use to validate the ILC algorithm for controlling the shape of the DM. The sketch of the experimental setup is shown in Fig. 1 .

We use coherent light from a semiconductor laser working at $\lambda=638 \mathrm{~nm}$. The light from the laser is then coupled with a single mode fiber. The other end of the fiber is placed in the focal point of the spherical lens L1 (focal length $f_{1}=100 \mathrm{~mm}$, diameter $\phi_{1}=1^{\prime \prime}$ ). The resulting collimated beam is folded by $90^{\circ}$ by the beam splitter (BS). The central part (9 $\mathrm{mm}$ in diameter) of the beam illuminates the clear aperture $(\phi=11 \mathrm{~mm})$ of the DM uniformly. The DM is a commercial membrane mirror with 48 actuators, produced by Adaptica Srl. The specification of this mirror can be found in [16]. The reflected light goes again through the BS in a relay system consisting of the lens L2 (focal length $f_{2}=250 \mathrm{~mm}$, diameter $\phi_{2}=2^{\prime \prime}$ ) and L3 (focal length $f_{3}=100 \mathrm{~mm}$, diameter $\left.\phi_{3}=2^{\prime \prime}\right)$. The purpose of the optical system L2-L3 is twofold. The first purpose is to optically conjugate the surface of the DM with the Shack-Hartmann WFS (S-H WFS, Thorlabs WFS S300-14AR, 1.3

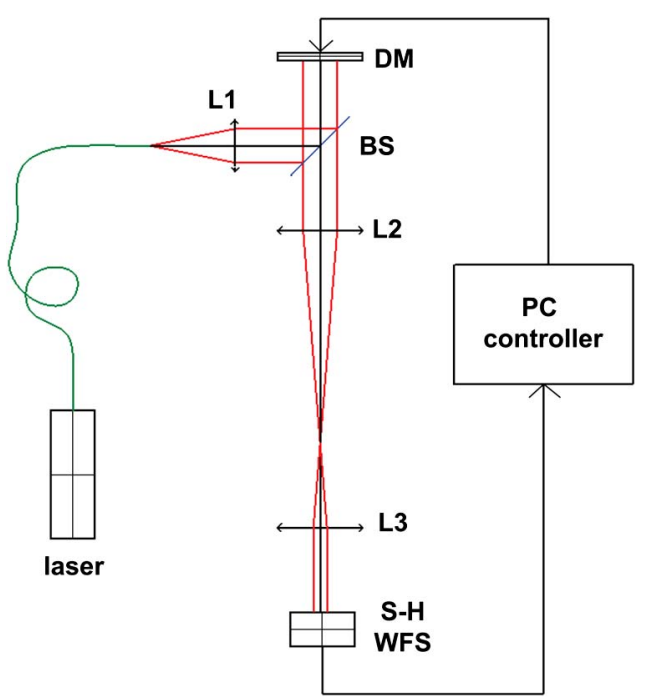

Fig. 1. Illustration of the experimental setup. 
Mpixel, $\lambda / 50 \mathrm{rms}$ accuracy). The optical system L2L3 also has the function to decrease the beam diameter by a factor $M=f_{3} / f_{2}$ that is needed for the $\mathrm{S}-\mathrm{H}$ WFS. The controller, that is implemented on a standard personal computer, receives measurements from the S-H WFS, and on the basis of these measurements sends a control signal to the DM. This feedback loop is established using a LabVIEW interface. The control algorithm is implemented in MATLAB.

The wavefront is sampled with the maximal sampling rate of the S-H WFS, which is $15 \mathrm{~Hz}$. The time constant of the membrane DM is in order of a few milliseconds [17]. Because the sampling period of the S-H WFS is much larger than a time constant of the DM, we are not able to observe the transient response of the DM using the S-H WFS. Consequently, in Section 3 we model the DM as a static system. The control sampling rate is $1 \mathrm{~Hz}$. Because the sampling rate of the S-H WFS is larger than the control sampling rate, in some degree we are able to reduce the effect of the measurement noise on the AO system. This is performed as follows. In between two consecutive control iterations, we take five measurement samples of the wavefront and we average these samples. This averaged wavefront is then used in the next control iteration.

\section{Iterative Learning Control for Membrane DM}

In this section we present an ILC algorithm for controlling the shape of a membrane DM. We study its stability and convergence rate. As a result of this study, we give a physical interpretation of the parameters of the ILC algorithm. This physical interpretation gives us guidelines for its (optimal) tuning.

In this paper, the wavefront that is produced by the DM will be represented using the Zernike polynomials basis expansion (the Malacra notation is used in [18]):

$$
\Phi(x, y) \approx \sum_{i=1}^{36} \alpha_{i} Z_{i}(x, y),
$$

where $\Phi(x, y)$ is the spatial distribution of the wavefront, $\alpha_{i}$ is the $i$ th coefficient of the Zernike polynomials expansion, and $Z_{i}(x, y)$ is the $i$ th Zernike polynomial. The steady-state model of the DM is given by $[\underline{13}, \underline{14}]$ :

$$
\mathbf{W}=F \mathbf{V},
$$

where $\mathbf{W} \in \mathbb{R}^{36}, \mathbf{W}=\left[\begin{array}{llll}\alpha_{1} & \alpha_{2} & \ldots & \alpha_{36}\end{array}\right]^{T}$ is the wavefront (membrane shape of the DM) expressed as Zernike coefficients, $F \in \mathbb{R}^{36 \times 48}$ is the influence matrix, and the vector $\mathbf{V} \in \mathbb{R}^{48}$ is given as follows:

$$
\mathbf{V}=\left[\begin{array}{llll}
u_{1}^{2} & u_{2}^{2} & \ldots & u_{48}^{2}
\end{array}\right]^{T},
$$

where $u_{i}$ is the control voltage applied to the $i$ th channel of the DM. As it can be seen from Eqs. (2) and (3), the model of the DM is a nonlinear

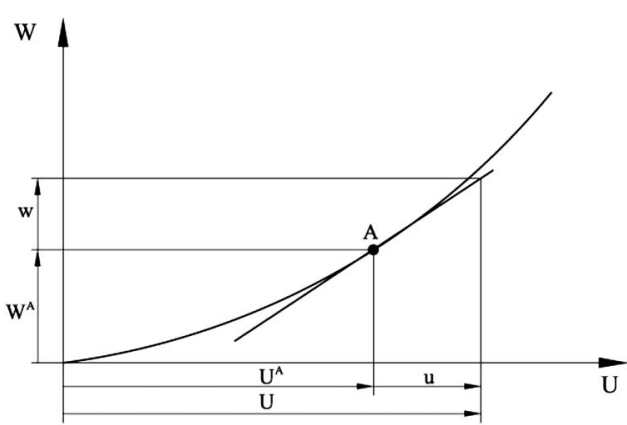

Fig. 2. Linear and nonlinear model of the DM.

(quadratic) function of the applied voltages. In contrast to $[13,14]$, in this paper we propose a control algorithm that is based on the linearized model of the DM. We identify a linear model of the DM, directly from experimental data, using the identification procedure explained in Section $\underline{4}$. By neglecting higher-order terms of the Taylor expansion, a linearized model of the DM around the working point $A$ can be written as (see Fig. $\underline{2}$ ):

$$
\begin{gathered}
\mathbf{w}=M \mathbf{u}, \\
\mathbf{u}=\mathbf{U}-\mathbf{U}^{A}, \quad \mathbf{w}=\mathbf{W}-\mathbf{W}^{A},
\end{gathered}
$$

where $\mathbf{U}=\left[\begin{array}{llll}u_{1} & u_{2} & \ldots & u_{48}\end{array}\right]^{T}$ is the vector of (total) voltages, $\mathbf{U}^{A}$ is the vector of working point voltages, $\mathbf{u}$ is the vector of relative voltages, $\mathbf{W}^{A}$ is the wavefront (membrane shape of the DM) produced by $\mathbf{U}^{A}, \mathbf{W}$ is the wavefront produced by $\mathbf{U}, \mathbf{w}$ is the relative wavefront, and $M$ is the influence matrix of the linear model. In this paper the working point voltages are chosen as follows:

$$
\mathbf{U}^{A}=\left[\begin{array}{llll}
u_{A} & u_{A} & \ldots & u_{A}
\end{array}\right]^{T},
$$

where $u_{A}$ is equal to $50 \%$ of the maximal voltages. It should be stressed that the model in Eqs. (4) and (5) depends on the working point voltages $\overline{\mathbf{U}}^{A}$. From Fig. 2, we can conclude that for different working point voltages, we obtain a different linear model of the DM. Furthermore, if $\mathbf{U}$ is "close" to $\mathbf{U}^{A}$, then the linear model accurately describes the behavior of the DM. On the other hand, if $\mathbf{U}$ is "far away" from $\mathbf{U}^{A}$, then the linear model is less accurate. However, as we experimentally demonstrate in Section 5 , the ILC algorithm can effectively handle these model uncertainties. For more details about robustness of the ILC algorithm with respect to model uncertainties, see $[19,20]$ and references therein.

Let the control iteration be denoted by $k$ (the control sampling rate is $1 \mathrm{~Hz}$ ). Further, let the wavefront (membrane shape of the DM), at the control iteration $k$, be denoted by $\mathbf{W}_{k}$. Similarly, we denote the relative membrane shape by $\mathbf{w}_{k}=\mathbf{W}_{k}-\mathbf{W}^{A}$. From Eqs. (4) and (5) it follows: 


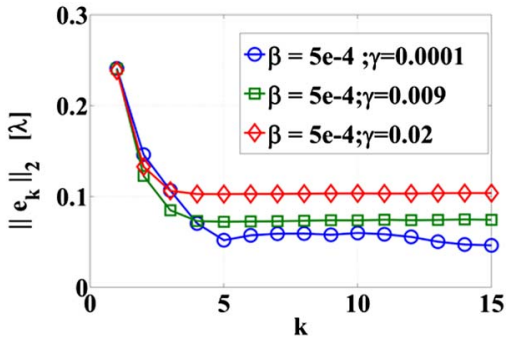

(a)

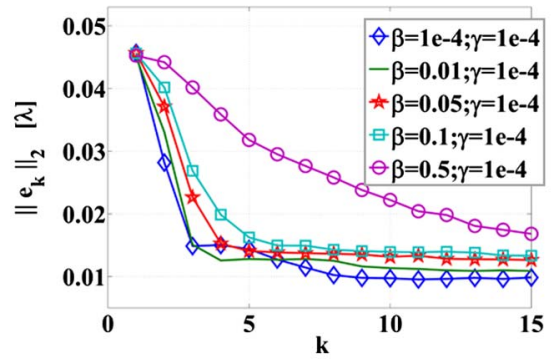

(c)

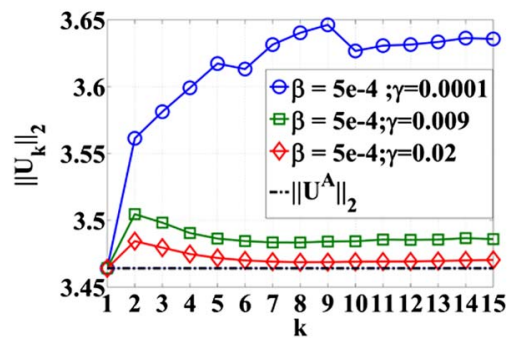

(b)

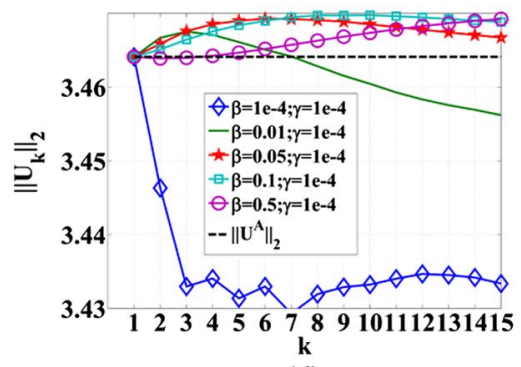

(d)

Fig. 3. Analysis of the convergence of the wavefront from an arbitrary to a flat wavefront, for different values of $\beta$ and $\gamma$. (a) Convergence of the norm of the tracking error for different $\gamma$. (b) Convergence of the norm of the total voltages for different $\gamma$. (c) Convergence of the norm of the tracking error for different $\beta$. (d) Convergence of the norm of the total voltages for different $\beta$.

$$
\mathbf{w}_{k}=M \mathbf{u}_{k}
$$

where $\mathbf{u}_{k}=\mathbf{U}_{k}-\mathbf{U}^{A}$ is the vector of relative voltages at the control iteration $k$, and $\mathbf{U}_{k}$ is vector of (total) voltages at control iteration $k$. The wavefront that is sensed by the S-H WFS, is the combination of the wavefront produced by actuating the DM and static wavefront aberrations initially present in the AO system [12]. These static wavefront aberrations come from nonflatness of the DM (when the voltages are not applied to DM) and from imperfections and misalignments of the optical components. Because the wavefront $\mathbf{W}_{k}$ corresponds to the membrane shape, this wavefront is obtained by subtracting all static wavefront aberrations from the wavefront measured by S-H WFS. As we explain in Section 5.A, the ILC algorithm can be easily modified such that it takes into account static wavefront aberrations of the AO system.

For simplicity, in this paper we did not include the measurement noise in Eq. (7). In reality the measured wavefront is corrupte $\bar{d}$ by the measurement noise of the S-H WFS. As we have explained in Section 2, the effect of the noise is reduced by averaging the wavefront between the two consecutive control iterations. Moreover, as it will be explained later, by adjusting the parameters of the ILC algorithm we are able to additionally increase noise immunity of the AO system.

The goal of the ILC algorithm is to produce a wavefront of the desired shape. Let such a wavefront be denoted by $\mathbf{W}_{d}$. The relative desired wavefront is denoted by $\mathbf{w}_{d}$ and it is defined as: $\mathbf{w}_{d}=\mathbf{W}_{d}-\mathbf{W}^{A}$. The wavefront error $\mathbf{e}_{k}$ at the control iteration $k$ is defined as follows:

$$
\mathbf{e}_{k}=\mathbf{w}_{d}-\mathbf{w}_{k}=\mathbf{w}_{d}-M \mathbf{u}_{k} .
$$

The error at the time instant $k+1$ is given by:

$$
\mathbf{e}_{k+1}=\mathbf{w}_{d}-M \mathbf{u}_{k+1} .
$$

From Eqs. (ㅁ) and (ㅁ) we have:

$$
\mathbf{e}_{k+1}=\mathbf{e}_{k}-M \Delta \mathbf{u}_{k},
$$

where

$$
\Delta \mathbf{u}_{k}=\mathbf{u}_{k+1}-\mathbf{u}_{k} .
$$

For a given $\mathbf{u}_{k}$ and $\mathbf{e}_{k}$, the optimal ILC law [20] is obtained by solving the following optimization problem:

$$
\min _{\mathbf{u}_{k+1}}\left\{\mathbf{e}_{k+1}^{T} Q_{e} \mathbf{e}_{k+1}+\mathbf{u}_{k+1}^{T} Q_{u} \mathbf{u}_{k+1}+\Delta \mathbf{u}_{k}^{T} Q_{\Delta u} \Delta \mathbf{u}_{k}\right\},
$$

where $Q_{e}, Q_{u}$, and $Q_{\Delta u}$ are the weighting matrices. The first term in Eq. (12) penalizes the wavefront error at the control iteration $k+1$. The second term penalizes the voltages for the control iteration $k+1$. Finally, the last term of the cost function Eq. (12) penalizes the difference between the voltages between the two consecutive control iterations, $k$ and $k+1$. In this paper we chose the weighting matrices of the cost function Eq. (12) as follows:

$$
Q_{e}=I, \quad Q_{u}=\gamma I, \quad Q_{\Delta u}=\beta I,
$$

where $\beta$ and $\gamma$ are positive real numbers. By solving Eq. (12) for the selection of the weighting matrices Eq. (13) we obtain the control law in the following form [20]: 


$$
\mathbf{u}_{k+1}=Q\left(\mathbf{u}_{k}+L \mathbf{e}_{k}\right),
$$

where

$$
\begin{gathered}
Q=\left(M^{T} M+\gamma I+\beta I\right)^{-1}\left(M^{T} M+\beta I\right), \\
L=\left(M^{T} M+\beta I\right)^{-1} M^{T} .
\end{gathered}
$$

At the initial iteration $k=0$, the vector of relative voltages $\mathbf{u}_{0}$ needs to be initialized. We choose $\mathbf{u}_{0}=0$, which corresponds to the vector of total voltages $\mathbf{U}=\mathbf{U}^{A}$.

The experimental tuning of the parameters $\beta$ and $\gamma$ of the ILC control algorithm Eqs. (14)-(16) might be time consuming and it might not guarantee optimal performance of the $\mathrm{AO}$ system. In order to simplify the tuning of the ILC algorithm, in the sequel we derive stability and monotonic convergence conditions for the ILC algorithm. Furthermore, we show how the steady-state tracking error and inputs depend on the parameters $\beta$ and $\gamma$. Based on these insights we give application oriented guidelines for the selection of $\beta$ and $\gamma$.

\section{A. Stability and Convergence Rate of the ILC Algorithm}

By substituting Eq. (ㅇ) in Eq. (14), we obtain:

$$
\mathbf{u}_{k+1}=Q(I-L M) \mathbf{u}_{k}+Q L \mathbf{w}_{d} .
$$

The stability and convergence rate of the ILC algorithm is primarily determined by the spectral properties of the matrix $Q(I-L M)$. In order to perform the stability and convergence rate analysis, we introduce the Singular Value Decomposition [21] of the full rank influence matrix $M$ :

$$
M=E_{1}[\Sigma \quad 0] E_{2}^{T},
$$

where $E_{1} \in \mathbb{R}^{36 \times 36}$ and $E_{2} \in \mathbb{R}^{48 \times 48}$ are unitary matrices, and the matrix $\Sigma \in \mathbb{R}^{36 \times 36}$ is a diagonal matrix of singular values: $\Sigma=\operatorname{diag}\left(\sigma_{1}, \sigma_{2}, \ldots, \sigma_{36}\right)$, where $\sigma_{1} \geq$ $\sigma_{2} \geq \ldots \geq \sigma_{36}>0$ are singular values. From Eqs. (15) and $(\underline{16})$ we have:

$$
Q(I-L M)=\left[M^{T} M+(\gamma+\beta) I\right]^{-1} \beta .
$$

Using the SVD Eq. (18), we can write Eq. (19) as follows:

$$
Q(I-L M)=E_{2}\left[\begin{array}{cc}
{\left[\Sigma^{2}+(\gamma+\beta) I\right]^{-1} \beta} & 0 \\
0 & \frac{\beta}{\gamma+\beta} I
\end{array}\right] E_{2}^{T} .
$$

The ILC algorithm is stable if $\|Q(I-L M)\|_{2}<1$, where $\|\cdot\|_{2}$ denotes the 2-norm. From Eq. (20) we conclude:

$$
\|Q(I-L M)\|_{2}=\frac{\beta}{\gamma+\beta}
$$

and consequently, we conclude that the ILC algorithm is stable if

$$
\frac{\beta}{\gamma+\beta}<1
$$

Because by definition $\beta>0$ and $\gamma>0$, from Eq. (22) we see that the ILC algorithm is always stable. However, a stable ILC algorithm does not necessarily imply a fast convergence of the control voltages. Due to this, in the sequel we study a convergence rate of the ILC algorithm.

We say that the ILC algorithm is monotonically convergent, if

$$
\left\|\mathbf{u}_{\infty}-\mathbf{u}_{k+1}\right\|_{2} \leq \nu\left\|\mathbf{u}_{\infty}-\mathbf{u}_{k}\right\|_{2},
$$

where $\mathbf{u}_{\infty}=\lim _{k \rightarrow \infty} \mathbf{u}_{k}$ and $0 \leq \nu<1$ is the convergence rate of the ILC algorithm. The smaller $\nu$ is, the faster is the convergence of the ILC algorithm and vice-versa. From Eq. (17) we have:

$$
\mathbf{u}_{\infty}=Q(I-L M) \mathbf{u}_{\infty}+Q L \mathbf{w}_{d} .
$$

Because $\gamma>0$, we have $\|Q(I-L M)\|_{2}<1$. This implies that the matrix $I-Q(I-L M)$ is invertible, and by solving Eq. (24) for $\mathbf{u}_{\infty}$ we obtain:

$$
\mathbf{u}_{\infty}=[I-Q(I-L M)]^{-1} Q L \mathbf{w}_{d} .
$$

By substituting Eqs. (15) and (16) in Eq. (25) we obtain:

$$
\mathbf{u}_{\infty}=\left(M^{T} M+\gamma I\right)^{-1} M^{T} \mathbf{w}_{d} .
$$

Equation (26) gives the value of the steady-state relative voltage vector. This equation helps us to estimate the steady state voltages for the desired wavefront $\mathbf{w}_{d}$, for the influence matrix $M$, and for a chosen parameter $\gamma$. Next we will show how the convergence rate of the ILC algorithm depends on the parameters $\beta$ and $\gamma$. From Eq. (25) we have:

$$
Q L \mathbf{w}_{d}=[I-Q(I-L M)] \mathbf{u}_{\infty} .
$$

Further we have:

$$
\mathbf{u}_{\infty}-\mathbf{u}_{k+1}=\mathbf{u}_{\infty}-Q(I-L M) \mathbf{u}_{k}-Q L \mathbf{w}_{d} .
$$

Substituting Eq. (27) in Eq. (28), we obtain:

$$
\mathbf{u}_{\infty}-\mathbf{u}_{k+1}=Q(I-L M)\left(\mathbf{u}_{\infty}-\mathbf{u}_{k}\right) .
$$

From the last expression, we obtain:

$$
\left\|\mathbf{u}_{\infty}-\mathbf{u}_{k+1}\right\|_{2} \leq\|Q(I-L M)\|_{2}\left\|\left(\mathbf{u}_{\infty}-\mathbf{u}_{k}\right)\right\|_{2} .
$$

From Eq. (20) we have $\|Q(I-L M)\|_{2}=\beta /(\beta+\gamma)$. This implies that the convergence rate of the ILC algorithm is given by:

$$
\nu=\frac{\beta}{\beta+\gamma} .
$$


From the last equation we can observe that by increasing $\gamma$ the convergence rate $\nu$ decreases. That is, by increasing $\gamma$ we have a faster convergence of the ILC algorithm (since smaller $\nu$ gives faster convergence of the ILC algorithm). In the sequel, we will show that by increasing $\gamma$ we decrease the steadystate relative voltages. This way, we can prevent actuator saturation. However, as we show in the sequel, the increase of $\gamma$ has a negative effect on the steady-state tracking error. From Eq. ()ㅡ we obtain:

$$
\mathbf{e}_{\infty}=\mathbf{w}_{d}-M \mathbf{u}_{\infty}
$$

By substituting Eq. (26) in Eq. (22) we obtain:

$$
\mathbf{e}_{\infty}=\left[I-M\left(M^{T} M+\gamma I\right)^{-1} M^{T}\right] \mathbf{w}_{d} .
$$

Equation (33) can help us to estimate the steadystate wavefront error for a desired wavefront, for the influence matrix $M$ and for the chosen value of the parameter $\gamma$. As we prove in Appendix $\underline{A}$, the steady-state relative input and the wavefront error are bounded as follows:

$$
\begin{aligned}
& \left\|\mathbf{u}_{\infty}\right\|_{2} \leq \frac{1}{2 \sqrt{\gamma}}\left\|\mathbf{w}_{d}\right\|_{2}, \\
& \left\|\mathbf{e}_{\infty}\right\|_{2} \leq \frac{\gamma}{\gamma+\sigma_{36}^{2}}\left\|\mathbf{w}_{d}\right\|_{2} .
\end{aligned}
$$

From the analysis presented in this section, we are able to give a physical interpretation of the design parameters $\beta$ and $\gamma$, and to draw the following guidelines for the tuning of the ILC algorithm.

\section{B. Physical Interpretation of the Parameters of the ILC} Algorithm and Guidlines for its Tuning

- From Eq. (34) we know that by increasing $\gamma$ we decrease the upper bound on the steady-state voltages. This physically means that by increasing $\gamma$ we can prevent the steady state voltages to saturate. At the same time, we know that by increasing $\gamma$ the ILC algorithm converges more rapidly.

- On the other hand, from Eq. (35) we see that by increasing $\gamma$ the upper bound on the steady state wavefront error increases. This physically means that by increasing $\gamma$ we degrade the accuracy of the wavefront correction/generation.

- From Eq. (35) we can deduce that when $\gamma \rightarrow 0$, $\mathbf{e}_{\infty} \rightarrow 0$. However, this will never be achieved in practice since this condition only holds when there are no model uncertainties (due to linearization, we always have model uncertainties). Furthermore, there is always measurement noise in the system.

- From Eqs. (26) and (33) we see that the parameter $\beta$ does not influence the steady-state input and the steady-state wavefront error. However, from Eq. (31) we have that by increasing $\beta$ the convergence rate of the ILC algorithm is slower and vice-versa. The parameter $\beta$ can also be used to regularize badly conditioned matrices $M$ and consequently to improve the noise immunity of the system [20,22].

In this section, we have derived the ILC algorithm by assuming that at each control iteration $k$ we are able to measure $\mathbf{w}_{k}$. That is, we have assumed that the ILC algorithm is applied online. However, in Section 5C. we will experimentally show that with only one initial measurement of the membrane shape, and by learning the control input offline using the ILC algorithm, we are able to achieve a relatively good performance of the AO system. Although the accuracy of the wavefront generation using this offline method is slightly worse than the online method, the offline method can produce a relatively small wavefront error in only one control iteration.

\section{Identification of the Influence Function}

In order to identify the linearized model of the DM around the working point A (see Fig. 2), we introduce the following matrices:

$$
P=\left[\begin{array}{llll}
\mathbf{u}_{1} & \mathbf{u}_{2} & \ldots & \mathbf{u}_{N}
\end{array}\right], \quad D=\left[\begin{array}{llll}
\mathbf{w}_{1} & \mathbf{w}_{2} & \ldots & \mathbf{w}_{N}
\end{array}\right] .
$$

In Eq. (36), $\mathbf{u}_{i} \in \mathbb{R}^{48}, i=1, \ldots, N$, is a vector of random relative voltages varying between $0 \%$ and $\pm 30 \%$ (30\% of relative voltages correspond to $80 \%$ of total voltages, since the working point $\mathrm{A}$ is chosen at $50 \%$ of total voltages) and $\mathbf{w}_{i}$ is the membrane shape produced by $\mathbf{u}_{i}$ and $N$ is a relatively large number (in our case $N=200$ ). The influence matrix $M$ is identified by solving the following least-squares optimization problem:

$$
\min _{M}\|D-M P\|_{F}^{2}
$$

where $\|\cdot\|_{F}$ denotes the Frobenius norm [23]. The random voltages ensure that $P$ has full row rank, so the solution of the optimization problem Eq. (37) is then given by:

$$
M=D P^{\dagger}
$$

where $P^{\dagger}=P^{T}\left(P P^{T}\right)^{-1}$ denotes the matrix pseudoinverse. The random voltages ensure that the mirror is persistently excited and that the crosscoupling between the channels is captured by the identified model. In order to determine $\mathbf{w}_{k}$ and consequently to define $D$, we need to know $\mathbf{W}^{A}$. That is, we need to know the membrane shape produced by the working point voltages. In theory, the wavefront $\mathbf{W}^{A}$ can be obtain by subtracting all static wavefront aberrations (originating from the experimental setup when the voltages are not applied to the DM) from the total measured wavefront [12]. However, by applying the vector of working point voltages $\mathbf{U}^{A}$ at different time instants $k$, and by subtracting all static wavefront aberrations from the measured wavefront, we will observe different $\mathbf{W}_{k}^{A}$. This is due to the measurement noise of the S-H WFS. We solve this problem 
using the following strategy from [23]: we apply $\mathbf{U}^{A}$ 30 times to DM. Each time we measure the corresponding wavefront and subtract all static wavefront aberrations. After that, we determine $\mathbf{W}^{A}$ by averaging wavefronts from these $N=30$ measurements (assuming that the effect of the hysteresis of the DM is not significant).

In Section 5C we will compare the dynamical behavior of the AO system with the dynamical behavior of the modeled AO system that is based on the identified model $M$.

\section{Experimental Results}

In the first part of this section we present experimental results that illustrate the influence of $\beta$ and $\gamma$ on the dynamical behavior of the $\mathrm{AO}$ system. In the second part we illustrate the ability of the AO system to generate/compensate some typical Zernike polynomial wavefront aberrations. In the third part we compare the identified model of the DM with experimental results. Furthermore, we show that by taking one initial wavefront measurement and by applying the ILC algorithm offline, we are able to achieve a good performance of the AO system. Finally, in the fourth part we compare the ILC algorithm with other control algorithms.

\section{A. Dynamical Behavior}

In Fig. 3 we present experimental convergence rates of the wavefront error and voltages for different values of $\beta$ and $\gamma$. In this experiment, our goal is to make the wavefront sensed by S-H WFS to be equal to a flat wavefront. In this case, the desired total wavefront (desired membrane shape) for the ILC algorithm is: $\mathbf{W}_{d}=-\mathbf{d}$, where $\mathbf{d}$ is the measurement of all static wavefront aberrations in the AO system when the voltages are not applied to the DM. From Fig. $\underline{3}$
Table 1. Desired Wavefront Details

\begin{tabular}{lccc}
\hline Desired Aberration & Zernike Index & P-V $[\lambda]$ & RMS $[\lambda]$ \\
\hline Astigmatism & $\alpha_{4}$ & 0.34 & 0.1 \\
Defocus & $\alpha_{5}$ & 0.45 & 0.1 \\
Trefoil & $\alpha_{6}$ & 0.45 & 0.08 \\
Coma & $\alpha_{7}$ & 0.45 & 0.08 \\
\hline
\end{tabular}

we see that by increasing $\gamma$ we decrease the norm of the steady-state voltages. That is, by adjusting $\gamma$ we can prevent actuator saturation. However, by increasing $\gamma$ we increase the steady-state wavefront error, that is, we degrade the accuracy of the wavefront correction. We can also see that the convergence speed of the ILC algorithm decreases as $\beta$ increases. These experimental results confirm the theoretical conclusions that we drew in Section 3.

\section{B. Performance of the AO System}

In order to demonstrate the performance of the $\mathrm{AO}$ system, we have generated several wavefronts described by different modes of the Zernike polynomial basis. The details of the desired wavefronts are listed in Table 1.

The results of the wavefronts convergence are given in Figs. 4-8. For the purpose of demonstrating the performance of the AO system, we have used: $\beta=$ 0.0005 and $\gamma=0.0001$. Since we wanted to boost the performance of the AO system, for coma generation (Fig. 8) we have allowed some of the channels to saturate (this can be avoided by increasing $\gamma$ ). As we can see from these figures, the ILC algorithm guarantees relatively good wavefront generation performance with a final RMS wavefront error of about $0.01 \lambda$ for all the considered cases.

Figures 4-8. (a) Convergence of the norm of the wavefront error $\left\|\mathbf{e}_{k}\right\|_{2}$. (b) Convergence of the total

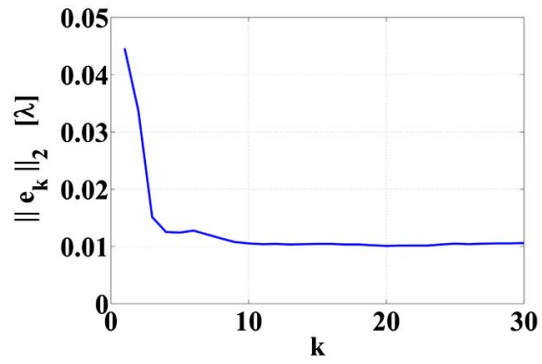

(a)

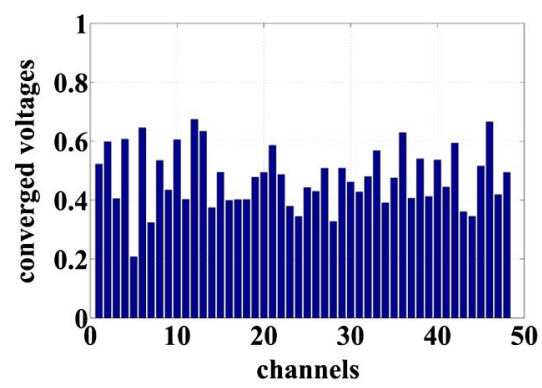

(c)

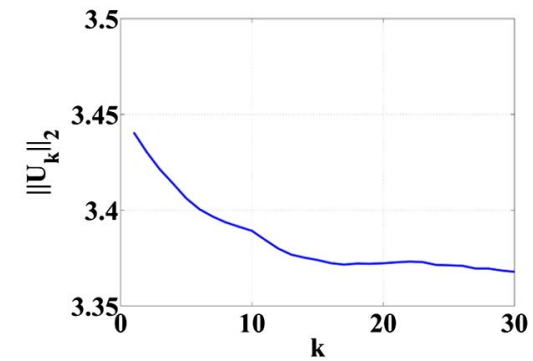

(b)

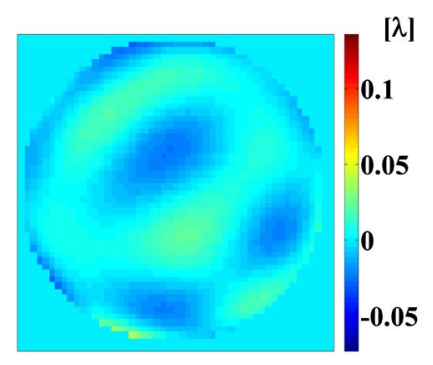

(d)

Fig. 4. Flat wavefront generation. 


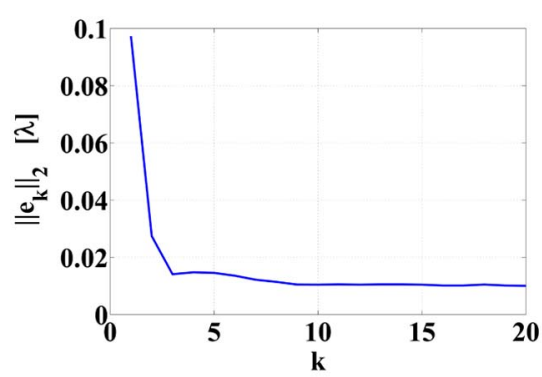

(a)

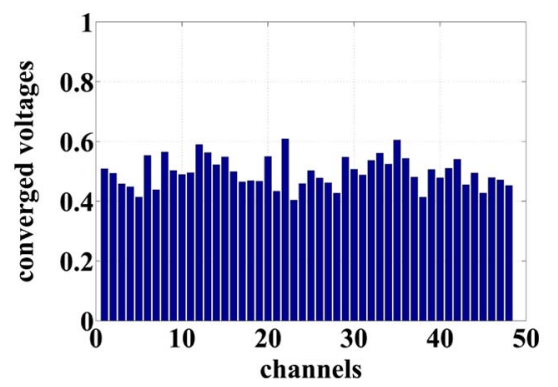

(c)

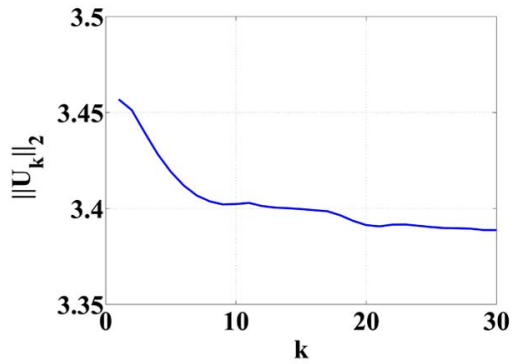

(b)

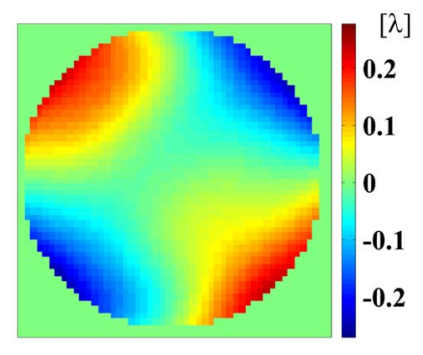

(d)

Fig. 5. Astigmatism generation.

voltages $\left\|\mathbf{U}_{k}\right\|_{2}$. (c) Converged voltages of each channel of the DM. (d) Converged wavefront.

\section{Comparison Between the Model and the Experimental Setup}

Using the identified influence function $M$ as a model of the DM, we have simulated the dynamical behavior of the AO system. We assume that the desired wavefront is a flat wavefront. We compare such a simulated behavior with the experimental results. The comparison is presented in Fig. 9 .

As it can be observed from Fig. 9 , the identified model of the influence function gives a relatively

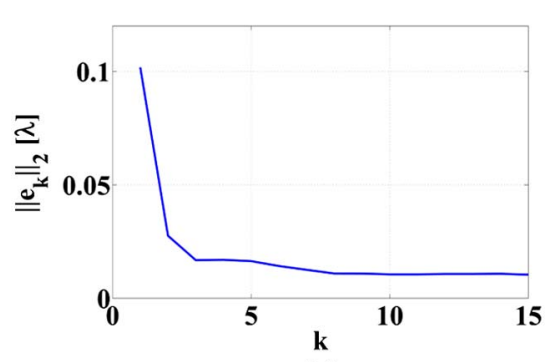

(a)

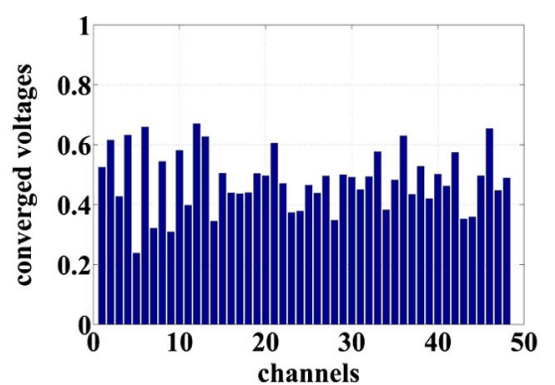

(c) good prediction of the dynamical behavior of the real AO system. This motivates us to perform the following experiment. For a desired wavefront equal to the flat wavefront and for a one initial measurement of the initial nonflatness of the DM, we run the ILC algorithm offline. That is, we run it without taking any additional measurements except the initial one. After the ILC algorithm has converged, we apply the converged voltages to the DM. The converged wavefront (steady-state wavefront) is given in Fig. 10. As comparison, we present a converged wavefront of the experiment when the ILC algorithm has been applied online.

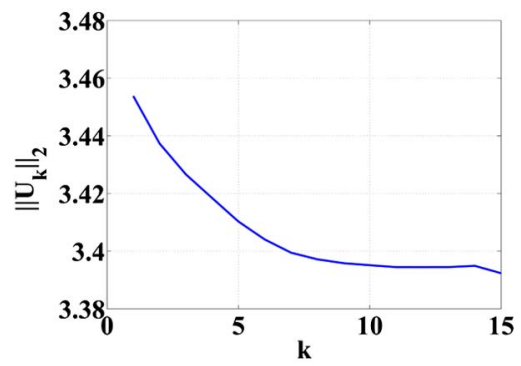

(b)

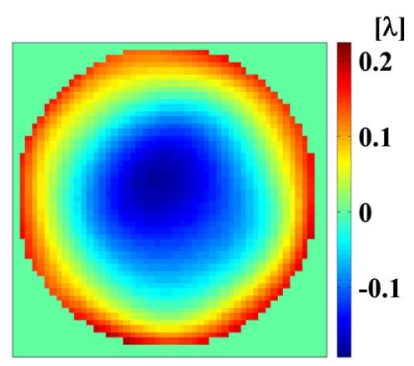

(d)

Fig. 6. Defocus generation. 


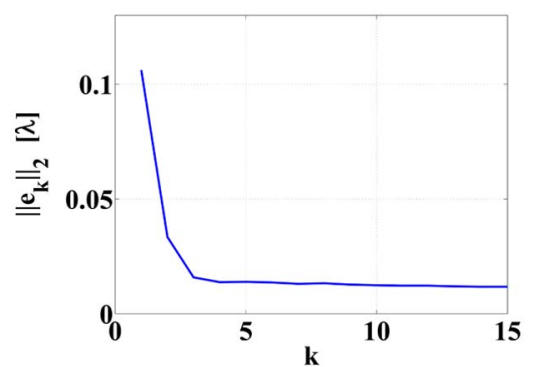

(a)

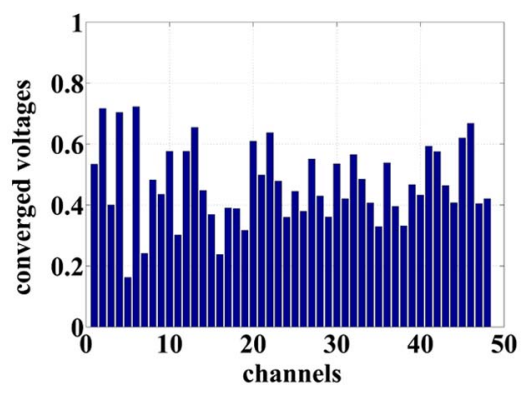

(c)

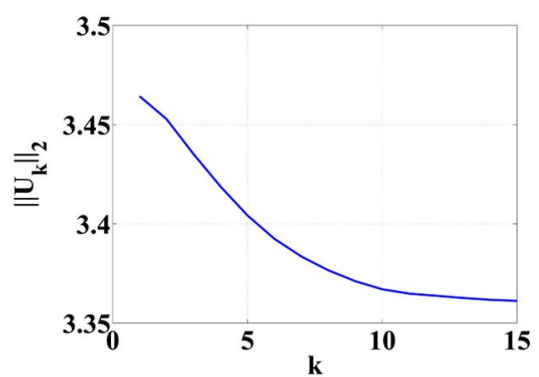

(b)

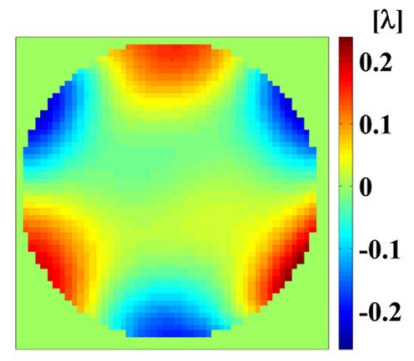

(d)

Fig. 7. Trefoil generation.

The RMS value of the steady state wavefront error when the ILC algorithm is performed offline is $0.015 \lambda$. On the other hand, the RMS value of the steady state wavefront error when the ILC algorithm is performed online is $0.011 \lambda$. These results shows us that using the identified model and the ILC algorithm, we can generate/correct wavefront aberrations with only one initial measurement of the wavefront.

\section{Comparison of ILC with Other Control Algorithms}

As a final demonstration of the advantages of the ILC algorithm, we compare it with a NNLS control

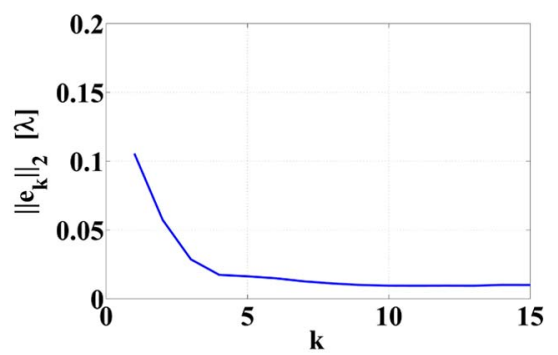

(a)

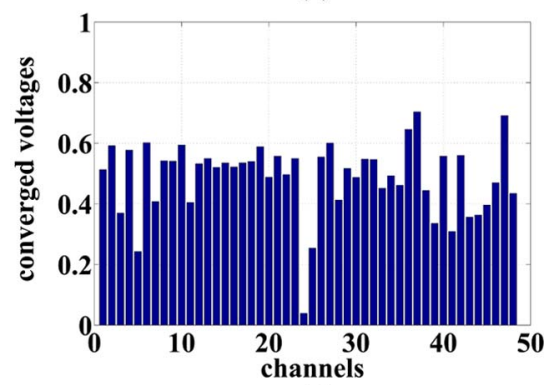

(c) algorithm of [13]. Furthermore, we compare the proposed ILC algorithm with the Steepest Descent ILC algorithm [20]:

$$
\mathbf{u}_{k+1}=\mathbf{u}_{k}+\alpha M^{T} \mathbf{e}_{k},
$$

where $\alpha=0.3$ is a step size. In this case the desired wavefront $W_{d}$ is Astigmatism $\alpha_{4}$ of $0.1 \lambda$ RMS. The results are presented in Fig. 11. In order to distinguish the proposed ILC algorithm from other algorithms, in this subsection we call it the optimal ILC algorithm. We have used $\beta=\gamma=0.0001$.

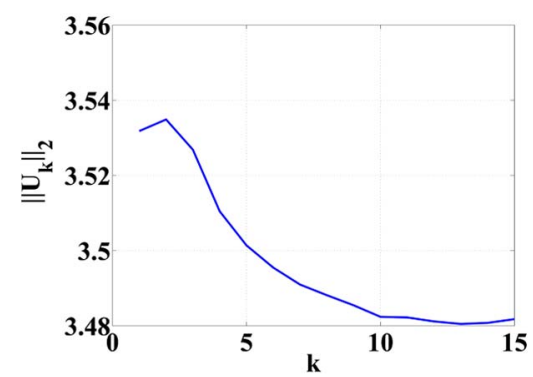

(b)

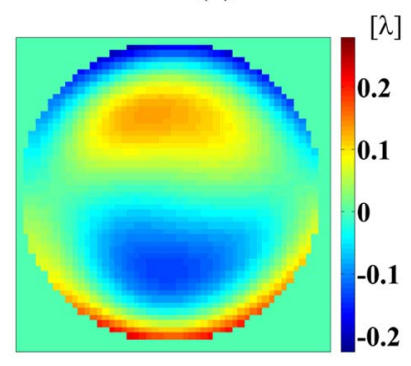

(d)

Fig. 8. Coma generation. 


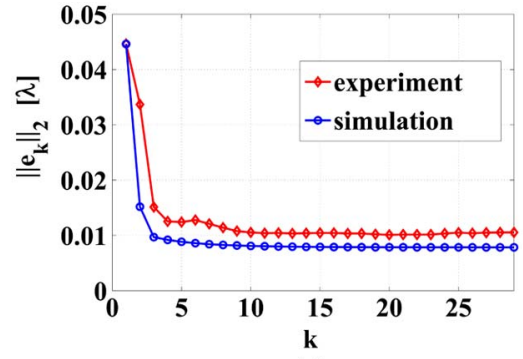

(a)

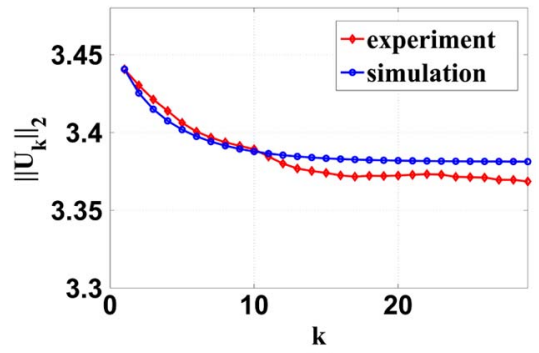

(b)

Fig. 9. Comparison of the model with the experimental results. (a) Convergence of the norm of the wavefront error $\left\|\mathbf{e}_{k}\right\|_{2}$. (b) Convergence of the norm of the total voltages $\left\|\mathbf{U}_{k}\right\|_{2}$.

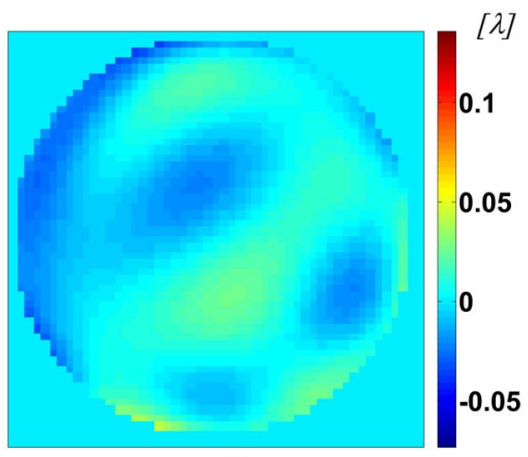

(a)

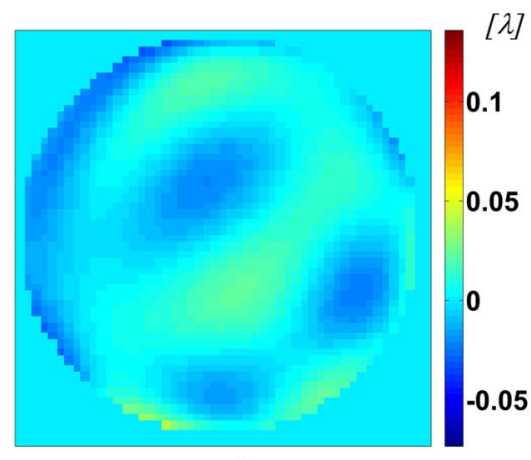

(b)

Fig. 10. Comparison of the offline with the online application of the ILC algorithm. (a) Converged wavefront when the ILC algorithm is applied offline. (b) Converged wavefront when the ILC algorithm is applied online.

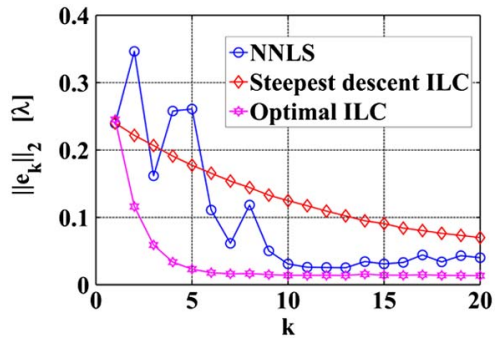

Fig. 11. Comparison of different control algorithms.

As it can be observed from Fig. 11, the optimal ILC algorithm (proposed in this paper) outperforms other two control algorithms. First of all, the optimal ILC algorithm converges in only 5 control iterations, while the NNLS converges after 10 iterations and Steepest descent ILC algorithm does not converge in 20 iterations. Next, the optimal ILC algorithm reaches the smallest value of the steady-state tracking error.

\section{Conclusion}

In this paper we have proposed an ILC algorithm for controlling the shape of a membrane DM. We have studied the stability and convergence rate of the novel algorithm and on the basis of this study we have given a physical interpretation of the controller parameters. This interpretation enabled us to derive a simple tuning procedure that in practice guarantees fast and stable convergence of the wavefront error. The experimental results show that by using the ILC algorithm we are able to achieve a relatively small value of the residual wavefront, while at the same time we are able to effectively control the saturation of voltages. Furthermore, the experimental results show that the ILC algorithm gives a small residual wavefront when it is applied offline with only one initial measurement of the membrane shape.

\section{Appendix A}

In this appendix we give a proof of Eqs. (34) and (35). Using Eq. (18) we can express Eqs. (26) and (3ㅜ) as follows:

$$
\begin{aligned}
& \mathbf{u}_{\infty}=E_{2} K E_{1}^{T} \mathbf{w}_{d}, \\
& \mathbf{e}_{\infty}=E_{1} S E_{1}^{T} \mathbf{w}_{d},
\end{aligned}
$$

where

$$
K=\left[\begin{array}{c}
\Sigma\left(\Sigma^{2}+\gamma I\right)^{-1} \\
0
\end{array}\right], \quad S=I-\Sigma^{2}\left(\Sigma^{2}+\gamma I\right)^{-1} .
$$

From Eqs. (1)-( $\underline{\mathrm{A} 3})$ we have:

$$
\left\|\mathbf{u}_{\infty}\right\|_{2} \leq\left\|E_{2} K E_{1}^{T}\right\|_{2}\left\|\mathbf{w}_{d}\right\|_{2},
$$




$$
\left\|\mathbf{e}_{\infty}\right\|_{2} \leq\left\|E_{1} S E_{1}^{T}\right\|_{2}\left\|\mathbf{w}_{d}\right\|_{2} .
$$

From Eq. (A3) we see that the singular values of $E_{2} K E_{1}^{T}$ have the following form:

$$
\frac{\sigma_{i}}{\sigma_{i}^{2}+\gamma}
$$

where $\sigma_{i}$ is the $i$ th singular value of $M$. The 2 -norm of $E_{2} K E_{1}^{T}$ is equal to its maximal singular value. Consider the function $f(x)=x /\left(x^{2}+\gamma\right)$, where $x$ is a real number. The maximum of $f(x)$ is equal to $1 /(2 \sqrt{\gamma})$ and it is achieved for $x=\sqrt{\gamma}$. When $x=\sigma_{i}, f\left(\sigma_{i}\right)$ is equal to Eq. (A6). From the above analysis, we have:

$$
\max _{\sigma_{i}} \frac{\sigma_{i}}{\sigma_{i}^{2}+\gamma} \leq \frac{1}{2 \sqrt{\gamma}} .
$$

When a singular value $\sigma_{i}$ is equal to $\sqrt{\gamma}$, then $\left\|E_{2} K E_{1}^{T}\right\|_{2}=\frac{1}{2 \sqrt{\gamma}}$. From Eqs. (4)-( $\underline{\mathrm{A} 7)}$ we obtain Eq. (34). From Eq. (A3) we see that the singular values of $E_{1} S E_{1}^{T}$ are given by:

$$
\frac{\gamma}{\sigma_{i}^{2}+\gamma}
$$

From Eq. (A8) we see that the maximal singular value of $E_{1} \overline{S E}_{1}^{T}$ (that is the 2-norm) is given by:

$$
\left\|E_{1} S E_{2}^{T}\right\|_{2}=\frac{\gamma}{\sigma_{36}^{2}+\gamma},
$$

where $\sigma_{36}$ is the minimal singular value of $M$. Using Eqs. (A5) and (A9) we obtain Eq. (35). This completes the proof.

This research is supported by the Dutch Ministry of the Economic Affairs and the Provinces of NoordBrabant and Limburg in the frame of the "Pieken in de Delta" program. The authors gratefully acknowledge the theoretical assistance of Jan van Neerven and also Roland Horsten and Rob Pols for their technical support.

\section{References}

1. F. Roddier, Adaptive Optics in Astronomy. (Cambridge, 1999).

2. R. K. Tyson, Principles of Adaptive Optics, 3rd ed., Series in Optics and Optoelectronics. (CRC, 2010).

3. M. J. Booth, "Adaptive optics in microscopy," Phil. Trans. R. Soc. A 365, 2829-2843 (2007).
4. J. Antonello, M. Verhaegen, R. Fraanje, T. van Werkhoven, H. C. Gerritsen, and C. U. Keller, "Semidefinite programming for model-based sensorless adaptive optics," J Opt. Soc. Am. A 29, 2428-2438 (2012).

5. A. Roorda, F. Romero-Borja, W. Donnelly III, H. Queener, T. Hebert, and M. Campbell, "Adaptive optics scanning laser ophthalmoscopy," Opt. Express 10, 405-412 (2002).

6. R. Zawadzki, S. Jones, S. Olivier, M. Zhao, B. Bower, J. Izatt, S. Choi, S. Laut, and J. Werner, "Adaptive-optics optical coherence tomography for high-resolution and high-speed 3D retinal in vivo imaging," Opt. Express 13, 8532-8546 (2005).

7. S.-W. Bahk, E. Fess, B. E. Kruschwitz, and J. D. Zuegel, "A high-resolution, adaptive beam-shaping system for highpower lasers," Opt. Express 18, 9151-9163 (2010).

8. T. Weyrauch, M. A. Vorontsov, J. Gowens, and T. G. Bifano, "Fiber coupling with adaptive optics for free-space optical communication," Proc. SPIE 4489, 177-184 (2002).

9. S. K. Ravensbergen, P. C. J. N. Rosielle, and M. Steinbuch, "Deformable mirrors with thermo-mechanical actuators for extreme ultraviolet lithography, design, realization and validation," Precision Engineering 37, 353-363 (2013).

10. S. K. Ravensbergen, "Adaptive Optics for Extreme Ultraviolet Lithography, Actuator Design and Validation for Deformable Mirror Concepts," PhD thesis, Technische Universiteit Eindhoven, 2012.

11. A. Polo, V. Kutchoukov, F. Bociort, S. F. Pereira, and H. P. Urbach, "Determination of wavefront structure for a Hartmann wavefront sensor using a phase-retrieval method," Opt. Express 20, 237-246 (2012).

12. L. Zhu, P. C. Sun, D. U. Bartsch, W. R. Freeman, and Y. Fainman, "Adaptive control of a micromachined continuousmembrane deformable mirror for aberration compensation," Appl. Opt. 38, 168-176 (1999).

13. S. Bonora and L. Poletto, "Push-pull membrane mirrors for adaptive optics," Opt. Express 14, 11935-11944 (2006).

14. E. Fernandez and P. Artal, "Membrane deformable mirror for adaptive optics, performance limits in visual optics," Opt. Express 11, 1056-1069 (2003).

15. A. Polo, A. Haber, S. F. Pereira, M. Verhaegen, and H. P. Urbach, "An innovative and efficient method to control the shape of push-pull membrane deformable mirror," Opt. Express 20, 27922-27932 (2012).

16. Adaptica Srl. Saturn user manual. http://www.adaptica.com/ site/en/pages/saturn.

17. G. Vdovin and P. M. Sarro, "Flexible mirror micromachined in silicon," Appl. Opt. 34, 2968-2972 (1995).

18. D. Malacara, Optical Shop Testing, volume 59, (Wiley, 2007).

19. A. Haber, R. Fraanje, and M. Verhaegen, "Linear computational complexity robust ilc for lifted systems," Automatica 48, 1102-1110 (2012).

20. D. A. Bristow, M. Tharayil, and A. G. Alleyne, "A survey of iterative learning control," Control Systems (IEEE, 2006) 26, 96-114.

21. G. H. Golub and C. F. Van Loan, Matrix Computations, 3rd ed. (Johns Hopkins, 1996).

22. A. Haber, P. R. Fraanje, and M. Verhaegen, "Fast and robust iterative learning control for lifted systems," World Congress 18, 3617-3622 (2011).

23. M. Verhaegen and V. Verdult, Filtering and System Identification, A Least Squares Approach (Cambridge, 2007). 